\title{
Water absorption by multifunctional protective epoxy composites
}

\author{
Vladimir Smirnov ${ }^{1, *}$ and Evgeniy Korolev ${ }^{1}$ \\ ${ }^{1}$ Moscow State University of Civil Engineering, Yaroslavskoe shosse, 26, Moscow, 129337, Russia
}

\begin{abstract}
Currently, thermoset-based polymer matrix composites are widely used in many areas of construction industry. Such composites demonstrate high compressive and flexural strength and are often characterized by high chemical stability under severe environmental conditions. One of the new promising areas of application of thermosetbased polymer matrix composites is the intumescent protective coating for metals; in normal conditions, such coatings can prevent the corrosion of metal constructions; during fire, expanded layer can prolong the time before critical failure. Water absorption is one of the primary characteristics of corrosion-protective materials; however, till now, there is only limited information about water absorption of multifunctional protective thermosetbased polymer matrix composites for intumescent coatings. In the present work, asymptotic values of water absorption of epoxy composites with polysiloxanes and fluorine-chlorine liquids are examined. The boundaries of control variables, within which the materials with required properties can be made, are determined. Silica, intercalated (expandable) graphite, and asbestos are used as fillers. Experiments were carried out in accordance with central composite experimental designs for full second-order regression models. It is shown that some admixtures can lead to notable decrease of water absorption.
\end{abstract}

\section{Introduction}

Steel is widely used in today construction industry. This is due to the fact that complex structures with sophisticated forms can be built quite quickly and at relatively low cost [1]. Steel can most often be found in bridge deck plates, high-rise buildings, etc. [3, 4].

However, there are at least to well known problems with ordinary steel. First problem is low corrosion resistance $[5,6]$. Second problem is that at high temperatures (open fire) elastic modulus of steel lowers down to inacceptable levels; this is the primary reason of failures of constructions during fire [7-10].

Both two mentioned problems with construction made of ordinary steel can be solved simultaneously by means of multifunctional coatings. During normal operation such coating can be used for protection from corrosion. During fire, intumescent coating transforms into expanded layer with low thermal conduction. This will prolong the time before critical failure of construction [11-13].

\footnotetext{
* Corresponding author: smirnov@nocnt.ru
} 
Currently, disperse filled epoxy matrix composites are widely used in many areas of construction industry. Such composited often characterized by high chemical stability under severe environmental conditions, including atmospheric effects. Epoxy matrix composites can also successfully be used for passive fire protection of steel [14-18].

Water absorption is one of the primary characteristics of corrosion-protective materials; however, till now there is only limited information about water absorption of multifunctional protective thermoset-based polymer matrix composites for intumescent coatings. In the present article we have examined both kinetics and asymptotic values of water absorption by epoxy polymers made with polysiloxanes and fluorine-chlorine liquids.

\section{Materials and methods}

As matrix material we have used DER-330 (Dow Chemical) epoxy resin. Silica (with specific surface equal to $200 \mathrm{~cm}^{2} / \mathrm{g}$ ) and asbestos fibers (AF) were used as non-intumescent fillers.

Intercalated (expandable) graphite is relatively new and very prospective intumescent material [19-22]. Being hydrophobic, expandable graphite (EG) can further decrease permeability of coating and increase the moisture resistance; some details about water resistance of intumescent composites with EG are already obtained [23]. In the present work EG with average flake diameter of $0.3 \mathrm{~mm}$ and $99 \%$ purity was used as intumescent admixture. Control samples were made with silica and AF, while intumescent samples were made with EG and AF.

To improve the rheology of compositions and decrease the water absorption we have additionally used two admixtures: polymethylphenylsiloxane (PMPS) and fluorine-chlorine liquids (FCL); latter were can also reduce the flammability of composite. Concentration of PMPS were varied on three levels - 0, 2 and 4\%. Concentration of FCL were varied on two levels -0 and $4 \%$.

During experiments two control variables were also varied:

- volumetric degree of filling $v_{f}$ (for control samples - from 0.2 to 0.6 , for intumescent samples - from 0.02 to 0.18 );

- relative amount of AF in mixture of fillers $c_{r}$ (for control samples - from 0 to 0.2 , for intumescent samples - from 0 to 0.65$)$. made:

Samples epoxy composites (EC) were of size $1 \times 1 \times 1 \mathrm{~cm}$. Four series of compositions were

- series 1: silica+AF+PMPS;

- series 2: silica $+\mathrm{AF}+\mathrm{FCL}$;

- series 3: EG+AF+PMPS;

- series 4: $\mathrm{EG}+\mathrm{AF}+\mathrm{FCL}$.

As a kinetic model of water absorption we have used the exponential dependence

$$
\delta M=\delta M_{\infty}\left(1-\exp \left(-\frac{t}{T_{1 / 2} \ln 2}\right)\right) .
$$

The parameters of this dependence are to follows.

1. The $\delta M$ is the current water absorption. This parameter can be directly determined from experimental data.

2. The $\delta M_{\infty}$ can be called asymptotic water absorption. It corresponds to experiment with infinite time.

3. The $T_{1 / 2}$ can be called half-time of saturation. This parameter characterizes speed of diffusion of water into sample.

Second and third parameters can be indirectly determined (fitted by means of least squares method) from experimental data. Within the framework of the open porosity model and with 
the assumption that the fluid is inert (saturation without destruction) these parameters characterize integral porosity and average pore size.

\section{Results and Discussion}

At the last stage of experiment values of $\delta M_{\infty}$ for all four series were approximated by quadratic polynomials in $\left(v_{f}, c_{r}\right)$ factor space. Obtained regression models are presented below.

For series 1:

$$
\begin{aligned}
& \delta M_{\infty,-1}=0.77+0.66 v_{f}+0.37 c_{r}+0.26 v_{f} c_{r}+0.07 v_{f}^{2}+0.19 c_{r}^{2} . \\
& \delta M_{\infty, 0}=0.61+0.22 v_{f}+0.22 c_{r}+0.17 v_{f} c_{r}-0.14 v_{f}^{2}+0.08 c_{r}^{2} . \\
& \delta M_{\infty, 1}=0.48+0.30 v_{f}+0.29 c_{r}+0.36 v_{f} c_{r}+0.12 v_{f}^{2}+0.12 c_{r}^{2} .
\end{aligned}
$$

For series 2:

$$
\begin{gathered}
\delta M_{\infty, 0}=0.72+0.39 v_{f}+0.26 c_{r}+0.11 v_{f} c_{r}-0.10 v_{f}^{2}+0.08 c_{r}^{2} . \\
\delta M_{\infty, 1}=0.86+0.39 v_{f}+0.11 c_{r}+0.05 v_{f} c_{r}-0.07 v_{f}^{2} .
\end{gathered}
$$

For series 3:

$$
\begin{gathered}
\delta M_{\infty,-1}=0.99+0.92 v_{f}+0.96 c_{r}+0.89 v_{f} c_{r}+0.36 c_{r}^{2} . \\
\delta M_{\infty, 0}=0.99+0.71 v_{f}+0.67 c_{r}+0.58 v_{f} c_{r}-0.09 v_{f}^{2}+0.17 c_{r}^{2} . \\
\delta M_{\infty, 1}=1.18+0.69 v_{f}+0.62 c_{r}+0.53 v_{f} c_{r}-0.08 v_{f}^{2} .
\end{gathered}
$$

For series 4:

$$
\begin{aligned}
& \delta M_{\infty, 0}=0.26+0.66 v_{f}+0.45 c_{r}+0.68 v_{f} c_{r}+0.49 v_{f}^{2}+0.24 c_{r}^{2} . \\
& \delta M_{\infty, 1}=0.49+0.74 v_{f}+0.73 c_{r}+0.84 v_{f} c_{r}+0.28 v_{f}^{2}+0.39 c_{r}^{2} .
\end{aligned}
$$

Indices of $\delta M_{\infty}$ in (1)-(10) reflects amount of admixture. For PMPS, "-1" corresponds to $0 \%$, " 0 " corresponds to $2 \%$ and " 1 " corresponds to $4 \%$. For FCL, " 0 " corresponds to $0 \%$ and " 1 " corresponds to $4 \%$.

Contour plots of asymptotic water absorption (regression models (1)-(10)) are presented on figures 1-10.

The water absorption of the unmodified EC in series 1 that is filled with silica and AF, corresponding to the center of the experimental design, is $0.77 \%$ (model (1)). The average water absorption calculated from the model in the entire factor space is

$$
\langle\delta M\rangle=\frac{1}{4} \int_{-1}^{1} d v_{f} \int_{-1}^{1} \delta M_{\infty,-1}\left(v_{f}, c_{r}\right) d c_{r} \approx 0.87 .
$$

This value is $29 \%$ higher than for unmodified EC filled with EG. This result is consistent with well-known hypothesis of reduced water absorption of EC based on hydrophobic filler. 


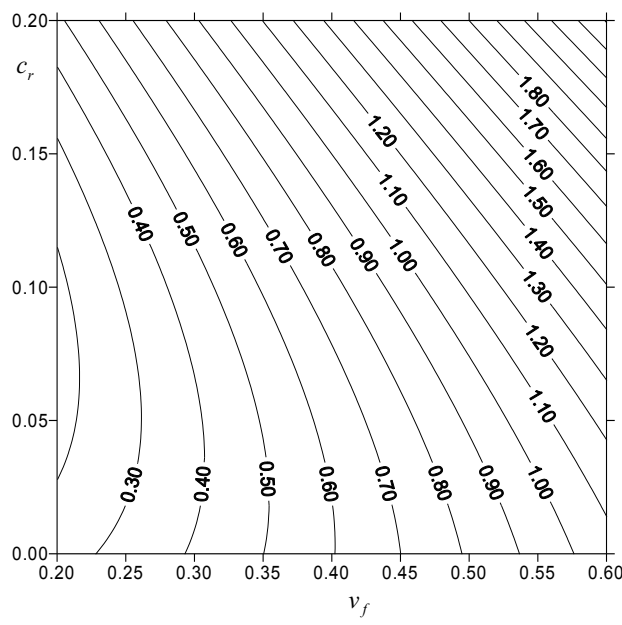

Fig. 1. Model (1).

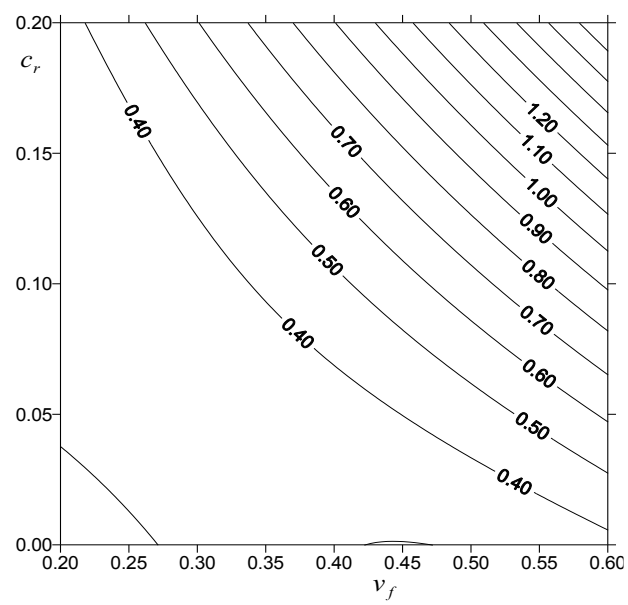

Fig. 3. Model (3).

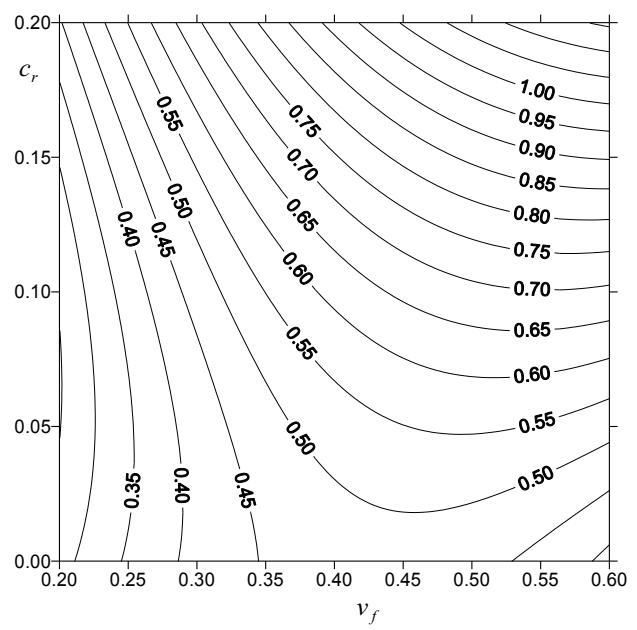

Fig. 2. Model (2).

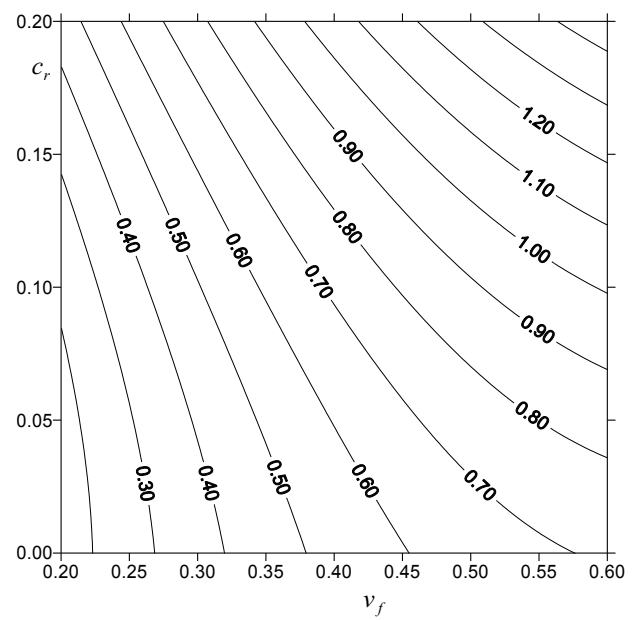

Fig. 4. Model (4).

During analysis, the joint effect of EC and AF should be taken into account. For EC with silica, the contour line that corresponds to the average absorption crosses the axis of the minimum AF content, whereas for EC with EG the same contour line crosses the axis of the maximum degree of filling (and it limits a significantly larger area). The average water absorption is associated with the influence of AF on the EC that is filled with EG. Unmodified samples corresponding to the right upper half of the factor space have almost unsatisfactory properties. The addition of AF to the unmodified EC with silica does not lead to such a significant change in water absorption.

Among the samples of EC with silica and AF, the best results are achieved for compositions which are modified by $2 \%$ and $4 \%$ PMPS. Despite the fact that the minimal water absorption corresponds to a composition with 2\% PMPS, it is advisable to use large concentrations. This is due to the fact that for composition with 2\% PMPS (model (2)) average absorption is $0.6 \%$, while for composition with $4 \%$ PMPS this value is only $0.55 \%$. Even this difference is quite small and comparable with measurement errors, the area that correspond to small water absorption for EC with 4\% PMPS (limited by contour lines 
$\delta M=0.4 \%$, figure 3) is larger than the same area for EC with $2 \%$ PMPS (limited by axes and contour lines $\delta M=0.4 \%$, figure 2).

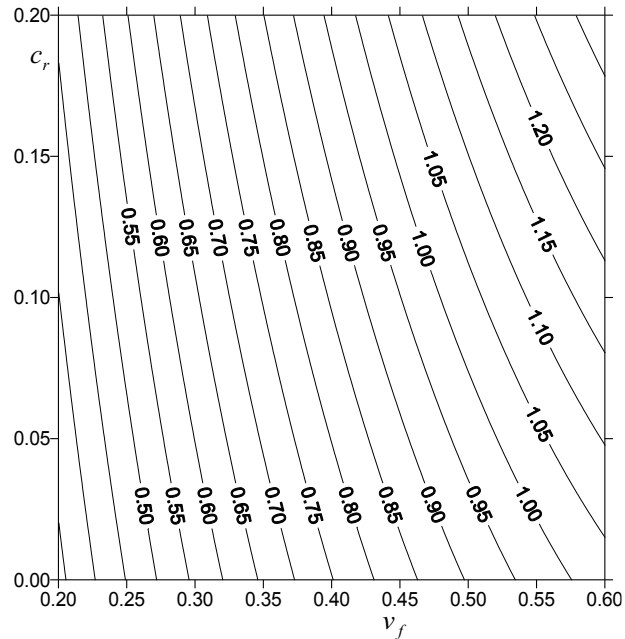

Fig. 5. Model (5).

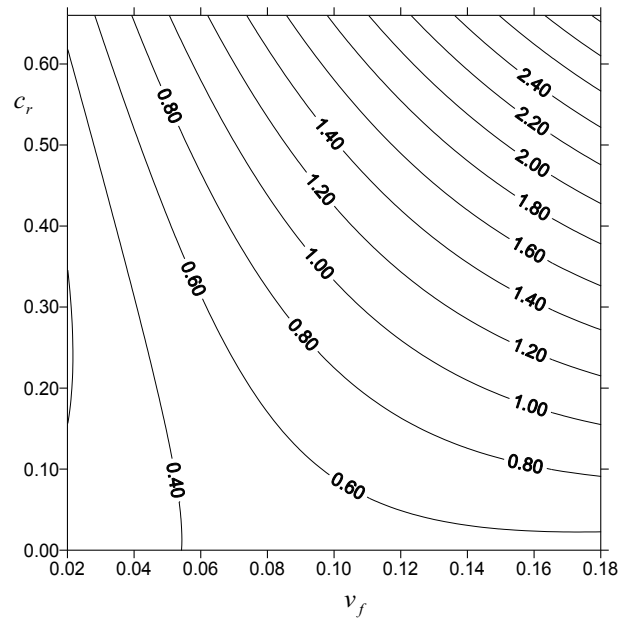

Fig. 7. Model (7).

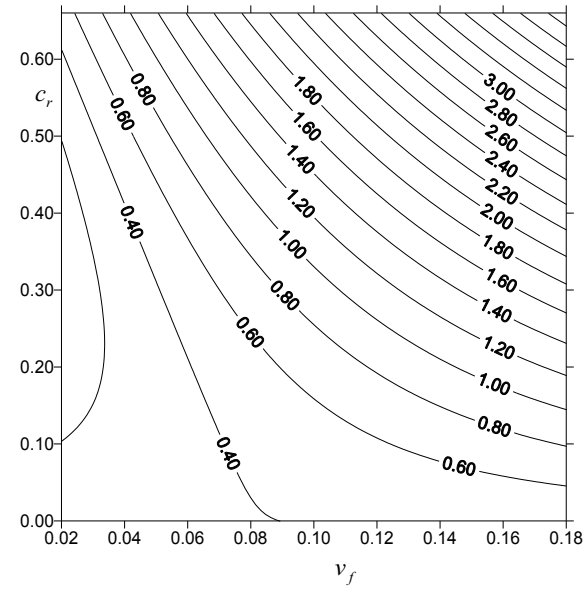

Fig. 6. Model (6).

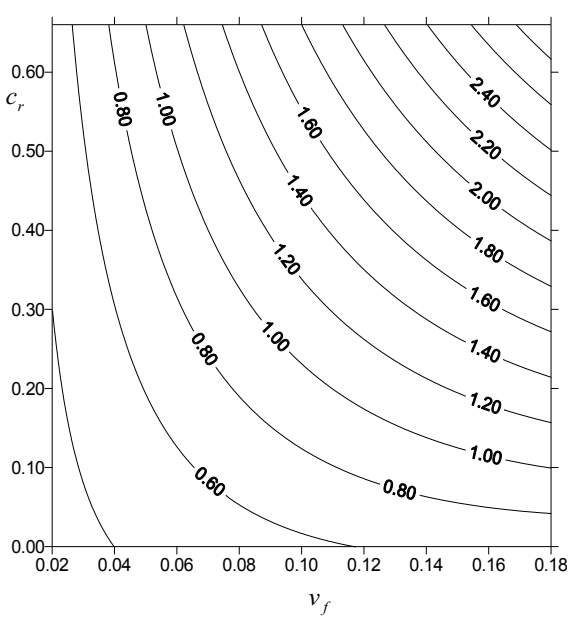

Fig. 8. Model (8).

The admixture of optimal amounts of PMPS in EC with silica allows not only to reduce the absolute value of water absorption, but also to reduce its dispersion in all factor space.

The water absorption of EC with silica and modified by $4 \%$ and, especially, by $2 \%$ of FCL, linearly increases with increasing of volumetric content of AF. Therefore, despite of a certain positive effect of modification by FCL, this additive can not be recommended for this type of EC.

In general, unmodified EC with EG and AF are of unsatisfactory water absorption. For EC with EG and AF, modification by FCL is preferred. Such modification reduces the average water absorption down to $0.2 \%$ and $0.7 \%$ for compositions with $2 \%$ and $4 \%$ of FCL, respectively. The area corresponding to the water absorption that is less than $0.2 \%$ is almost as large as half of the factor space (figure 9). 


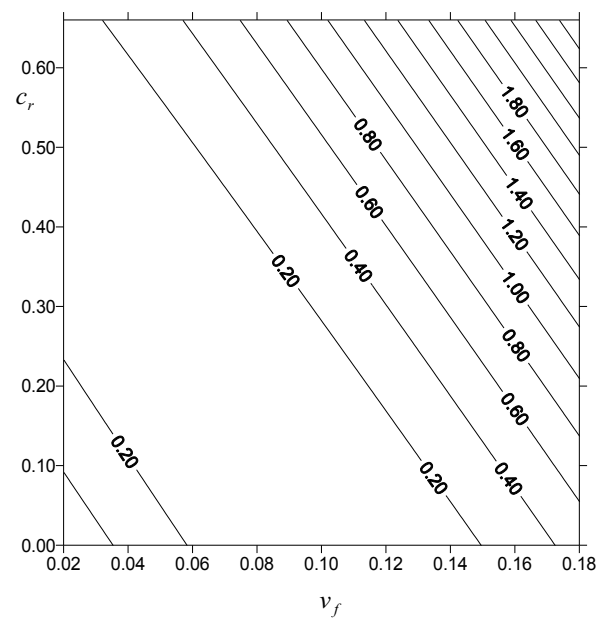

Fig. 9. Model (9).

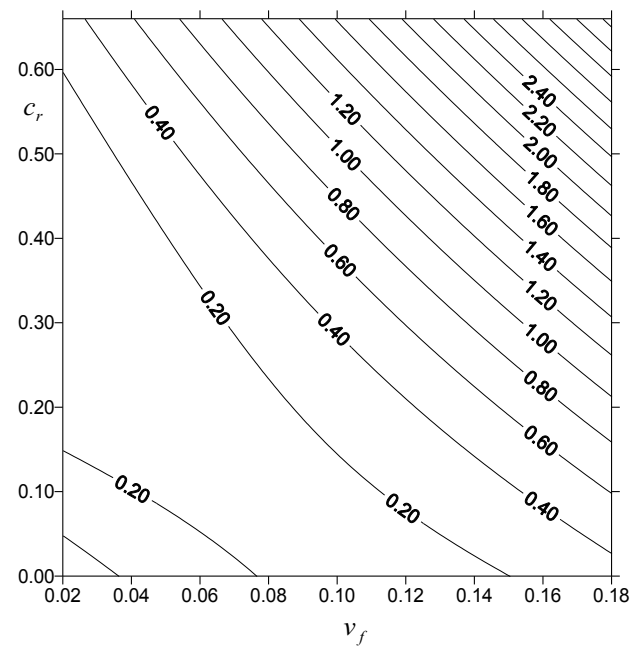

Fig. 10. Model (10).

Unfortunately, contour lines of equal water absorption (figures 9 and 10) indicates that for the series 4 of EC, the most effective way to reduce water absorption is to reduce both amount of $\mathrm{AF}$ and amount of EG.

In the present work the relatively low values of water absorption were obtained. This is due to the fact that only the surface layers of the material were saturated. The time of the experiment was only one month, while calculated half time of saturation is $60 \ldots 120$ days.

\section{Summary}

Admixtures of functional disperse phases in epoxy matrix composites can make such composites multifunctional. At the same time, such admixtures can cause negative effects on some properties.

It the present work we have studied the influence of several control variables to the water absorption of multifunctional epoxy matrix composites for intumescent coatings. It was shown that, in general, increase in volumetric degree of filling leads to the increase of water absorption. Still, some admixtures, including PMPS and FCL, allow to control the water absorption even for high degree of filling.

\section{Acknowledgments}

This work is supported by Ministry of Education and Science of Russian Federation, project "Theoretical and experimental models of functional composites based on prime nanomaterials" $7.6250 .2017 / 8.9$

\section{References}

1. U.K. Ghosh. Design and Construction of Steel Bridges. Singapore: Taylor \& Francis Group (2006)

2. J. Durkee. Steel Bridge Construction. Structural Engineering Handbook. Boca Raton: CRC Press (1999)

3. N.R.Baddoo. Journal of Constructional Steel Research, 64 (2008) 
4. C.G. Salmon et al. Steel Structures: Design and Behavior. New Jersey: Prentice Hall (2008)

5. L.L. Sheir. Corrosion: Metal/Environment Reactions. Oxford: Newnes-Butterworth (1976)

6. N.R. Short, J.K. Dennis. Transactions of the IMF, 75 (1997)

7. T.C.H Liu, M.K. Fahad, J.M. Davies. Journal of Constructional Steel Research, 58 (2002)

8. F. Wald, L. Simões da Silva, D.B. Moore, T. Lennon, M. Chladná, A. Santiago, M. Beneš, L. Borges. Fire Safety Journal, 41 (2006)

9. S.R. Najjar, I.W. Burgess. Engineering Structures, 18 (1996)

10. M. Feng, Y.C. Wang, J.M. Davies. Fire Safety Journal, 38 (2003)

11. M. Jimenez, S. Duquesne, S. Bourbigot. Thermochimica acta, 449 (2006)

12. B.K. Cirpici, Y.C. Wang, B.D. Rogers, S Bourbigot. Polymer Engineering \& Science, 56 (2016)

13. M. Jimenez, S. Duquesne, S. Bourbigot. Surface and coatings Technology, 201 (2006)

14. M. Jimenez, S. Bellayer, B. Revel, S. Duquesne, S. Bourbigot. Industrial \& Engineering Chemistry Research, 52 (2013)

15. J.S. Wang, Y. Liu, H.B. Zhao, J. Liu, D.Y. Wang, Y.P. Song. Y.Z.Wang. Polymer Degradation and Stability, 94 (2009)

16. C. Gérard, G. Fontaine, S. Bellayer, S. Bourbigot. Polymer Degradation and Stability, 97 (2012)

17. C. Gérard, G. Fontaine, S. Bourbigot. Polymers for Advances Technologies, 22 (2011)

18. M. Gomez-Mares, A. Tugnoli, G. Landucci, F. Barontini, V. Cozzani. Journal of Analytical and Applied Pyrolysis, 97 (2012)

19. S. Ullah, F. Ahmad. Polymer Degradation and Stability, 103 (2014)

20. C. Chiang, S. Hsu. Polymer International, 59 (2010)

21. J. Gu, G. Zhang, S. Dong, Q. Zhang, J. Kong. Surface and Coatings Technology, 201 (2007)

22. S. Yang, J. Wang, S. Huo, M. Wang, J. Wang, B. Zhang. Polymer Degradation and Stability, 128 (2016)

23. Z. Wang, E. Han, W. Ke. Corrosion Science, 49 (2007) 